Supporting information

\title{
Heterogeneous uptake of formic acid and acetic acid on mineral dust and coal fly ash
}

\author{
Yidan Wang ${ }^{\dagger,}$, Li Zhou ${ }^{\Perp}$, Weigang Wang ${ }^{*}, \dagger$, Maofa Ge ${ }^{*, \dagger, \ddagger, \S}$
}

${ }^{\dagger}$ State Key Laboratory for Structural Chemistry of Unstable and Stable Species, Beijing National

Laboratory for Molecular Sciences (BNLMS), CAS Research/Education Center for Excellence in Molecular Sciences, Institute of Chemistry, Chinese Academy of Sciences, Beijing 100190, P. R. China

${ }^{\ddagger}$ University of Chinese Academy of Sciences, Beijing 100049, P. R. China

\|National Engineering Research Center for Flue Gas Desulfurization, Department of Environmental Science and Engineering, Sichuan University, Chengdu, 610065, China.

${ }^{\S}$ Center for Excellence in Regional Atmospheric Environment, Institute of Urban Environment, Chinese Academy of Sciences, Xiamen, 361021, P. R. China

*E-mail: wangwg@iccas.ac.cn; gemaofa@iccas.ac.cn 
Figure S1, S10 and S11 show the SEM and TEM picture of authentic samples. Figure S2 - S5 show the XRD spectrum of the mineral dust particles and CFA and their mineralogy phases. Uptake curves of formic acid and acetic acid on authentic samples are shown in Figure S6-S9.The element mass percentage of authentic samples and correlation analysis of elements with uptake coefficient of monocarboxylic acid are listed in Table S1-S4.

\section{The correlation coefficient between the uptake coefficient and Na mass fraction (Table S5)}

XRD results show that IMD and XJS have an amount of albite. However, as the XRF method couldn't obtain the mass percentage of Na. The relative mass content was measured using TEM coupled with EDS (Energy Dispersive Spectrometer). The average mass percentage of Na at about three sites in the vision of TEM is $3.15 \%, 1.41 \%, 2.82,0.59 \%$ for IMD, XJS, ATD, CFA, respectively. The result is the relative mass fraction of element $\mathrm{Na}, \mathrm{Mg}, \mathrm{Al}, \mathrm{Si}, \mathrm{P}, \mathrm{S}, \mathrm{Cl}, \mathrm{K}, \mathrm{Ca}, \mathrm{Ti}$, $\mathrm{Mn}, \mathrm{Fe}, \mathrm{Cu}, \mathrm{Zn}$, which are not comparable with the results from XRF method. The correlation coefficient between the uptake coefficient and Na content was obtained as shown in Table S5. The results show a positive correlation indicate physical adsorption probably on albite though the correlation is not strong as $\mathrm{K}$. This result shows different mineral phase in the solid sample has different adsorption properties. Feldspar is an important aluminosilicate, and there are kinds of feldspar in different mineral dust samples. Further research of the adsorption behavior of acids on feldspar, such as albite and orthoclase et al., and other aluminosilicates under dry and humidity are needed which would help understanding acid adsorption on mineral dust samples. The uptake for formic acid and acetic on mineral dust increase with the content of the orthoclase and have a positive correlation with the content of the Si element. 


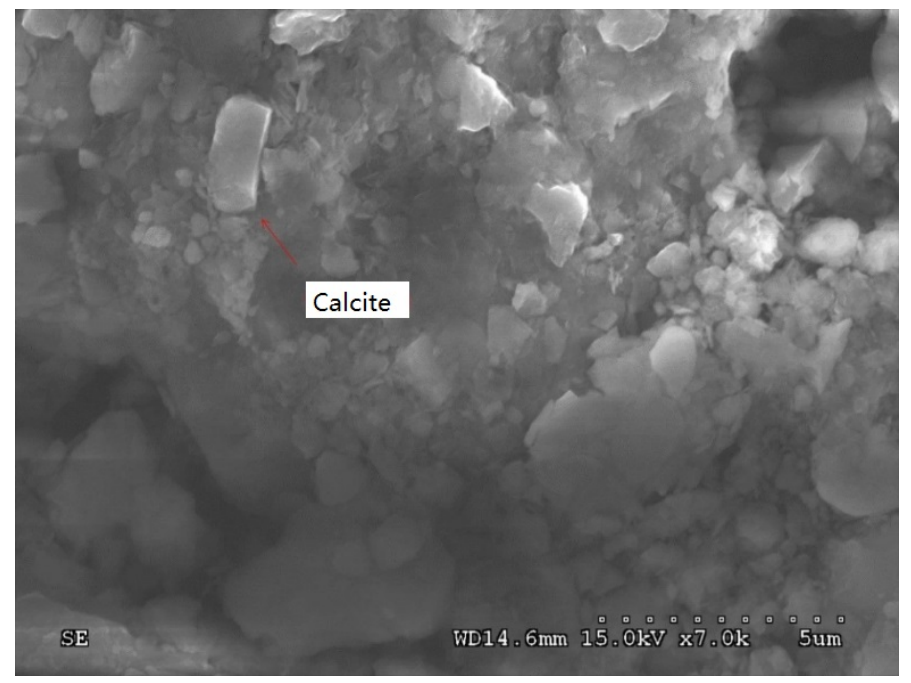

Figure S1. SEM image of Xinjiang sierozem mineral dust at $5 \mu \mathrm{m}$ level.

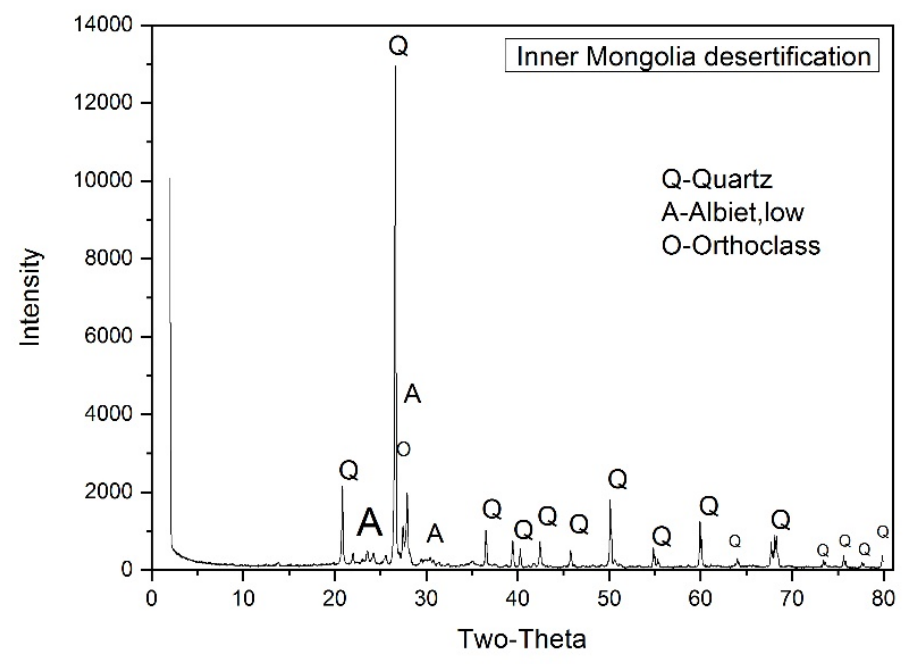

Figure S2. XRD spectrum of Inner Mongolia desertification. 


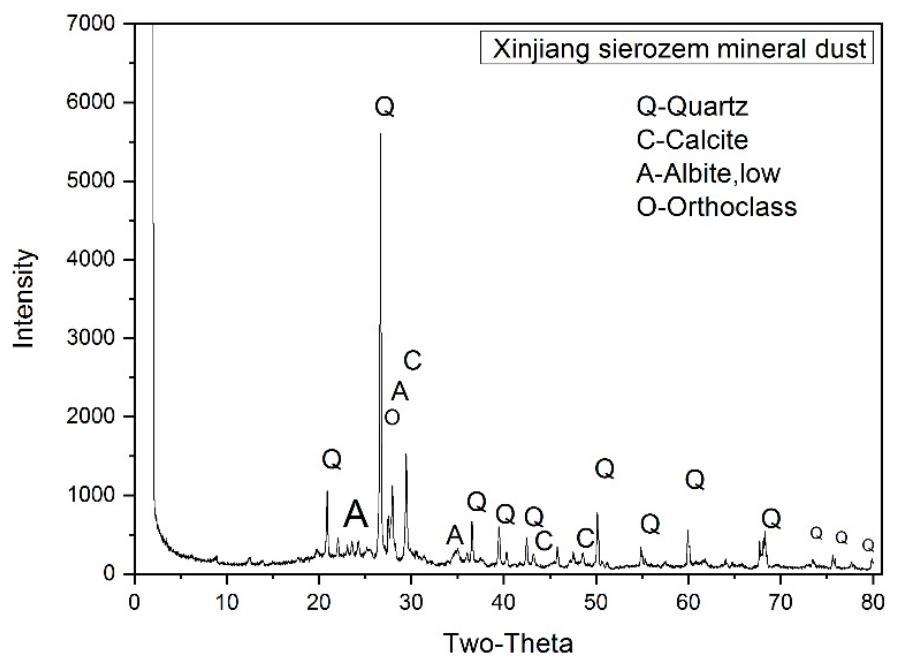

Figure S3. XRD spectrum of Xinjiang sierozem.

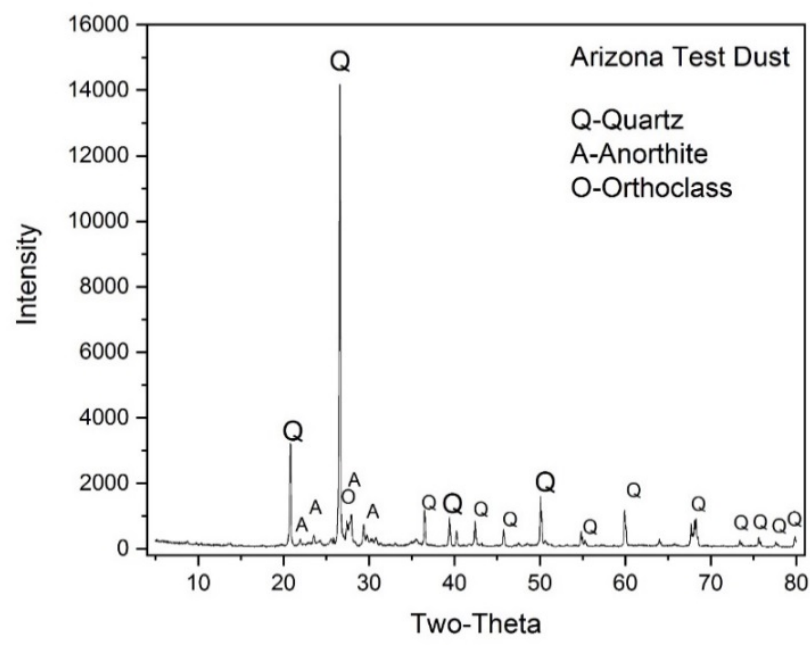

Figure S4. XRD spectrum of Arizona Test Dust. 


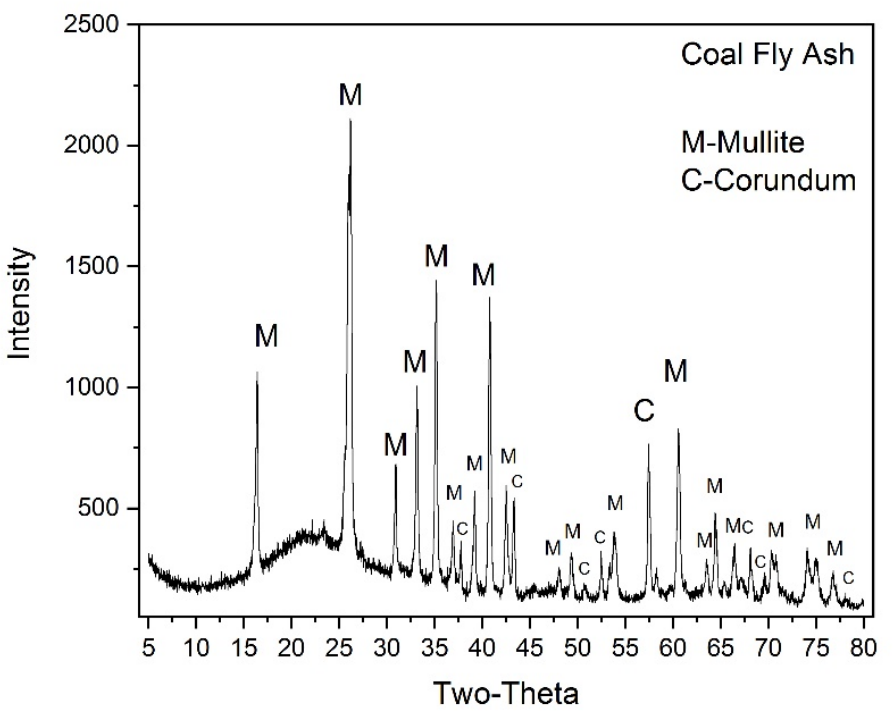

Figure S5. XRD spectrum of Coal Fly Ash. 

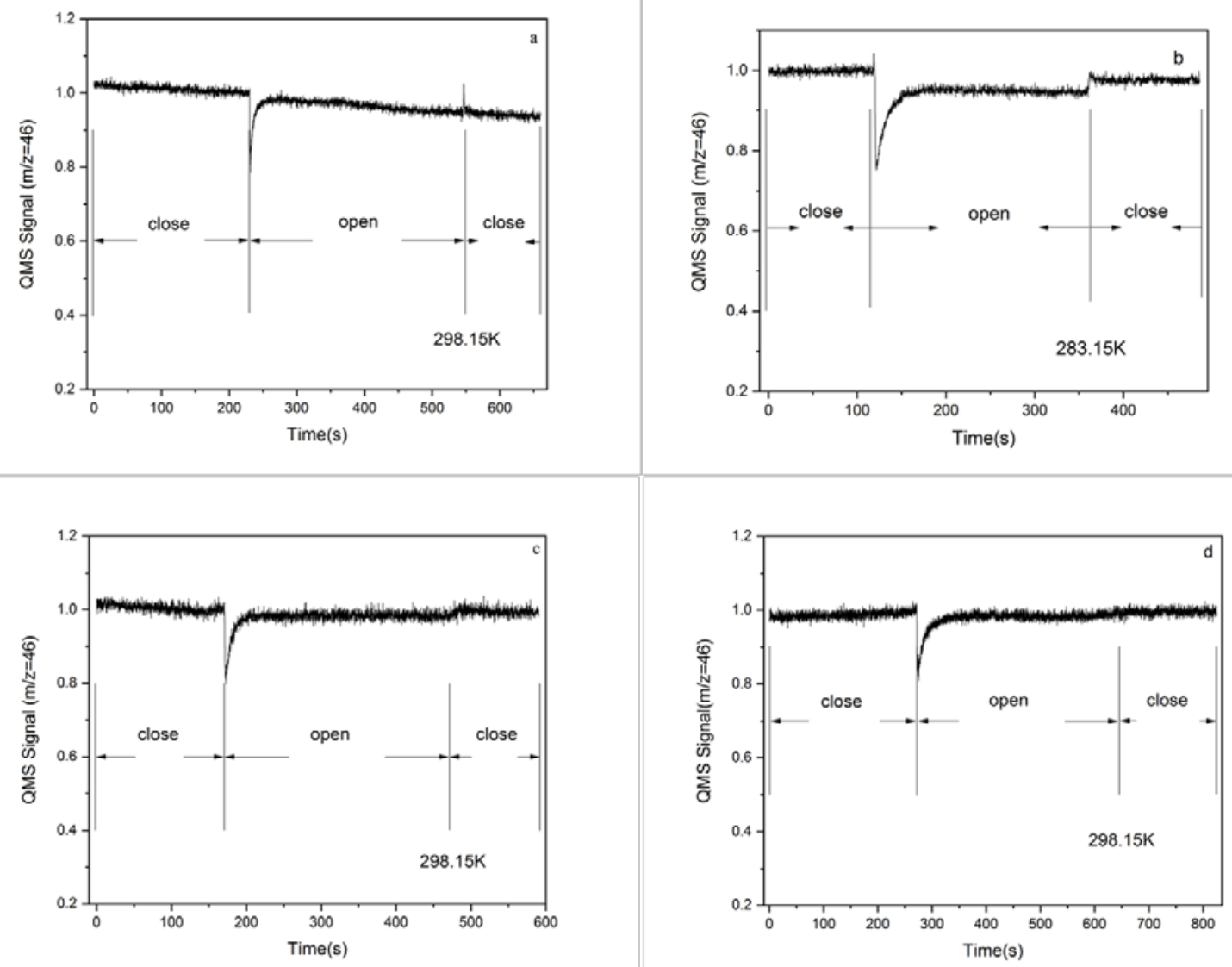

Figure S6. Uptake curves of formic acid on authentic samples (a)Inner Mongolia desertification (b)Xinjiang sierozem mineral dust (c) Arizona Test Dust (d) Coal Fly Ash 

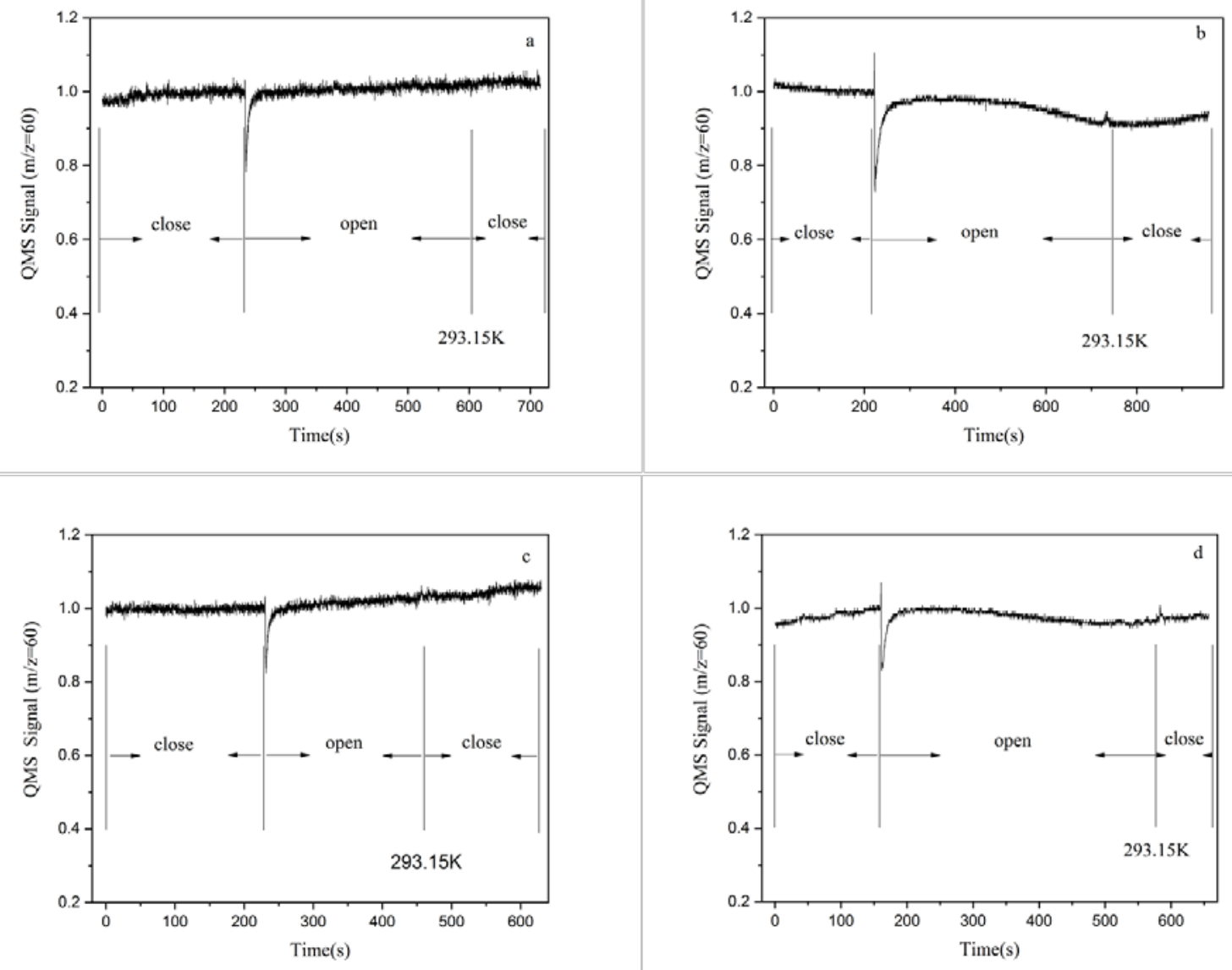

Figure S7. Uptake curves of acetic acid on authentic samples (a)Inner Mongolia desertification (b)Xinjiang sierozem mineral dust (c) Arizona Test Dust (d) Coal Fly Ash 

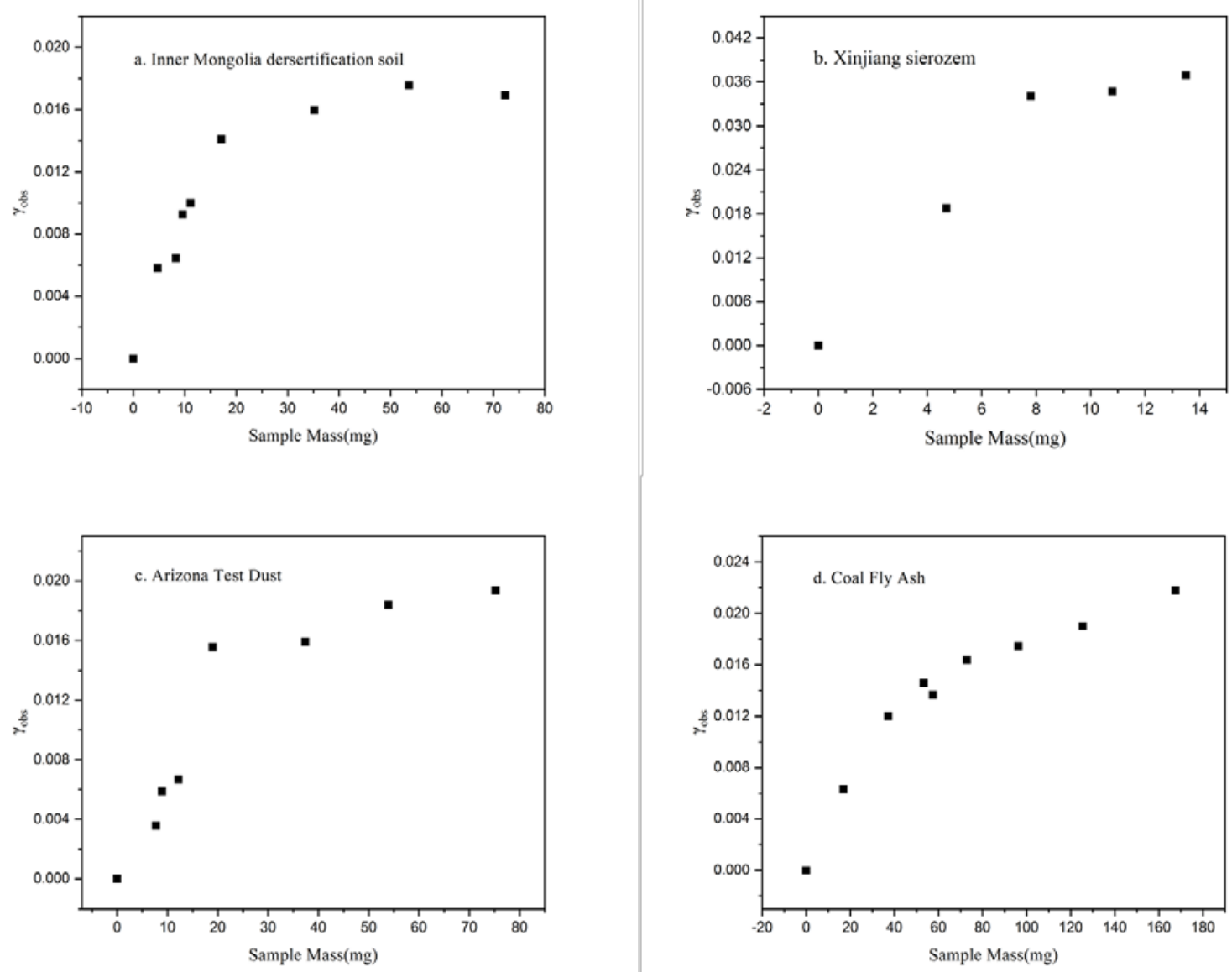

Figure S8. Linear interval comparation of a, Inner Mongolia desertification soil; b, Xinjiang sierozem; c, Arizona Test Dust; d, Coal Fly Ash. 

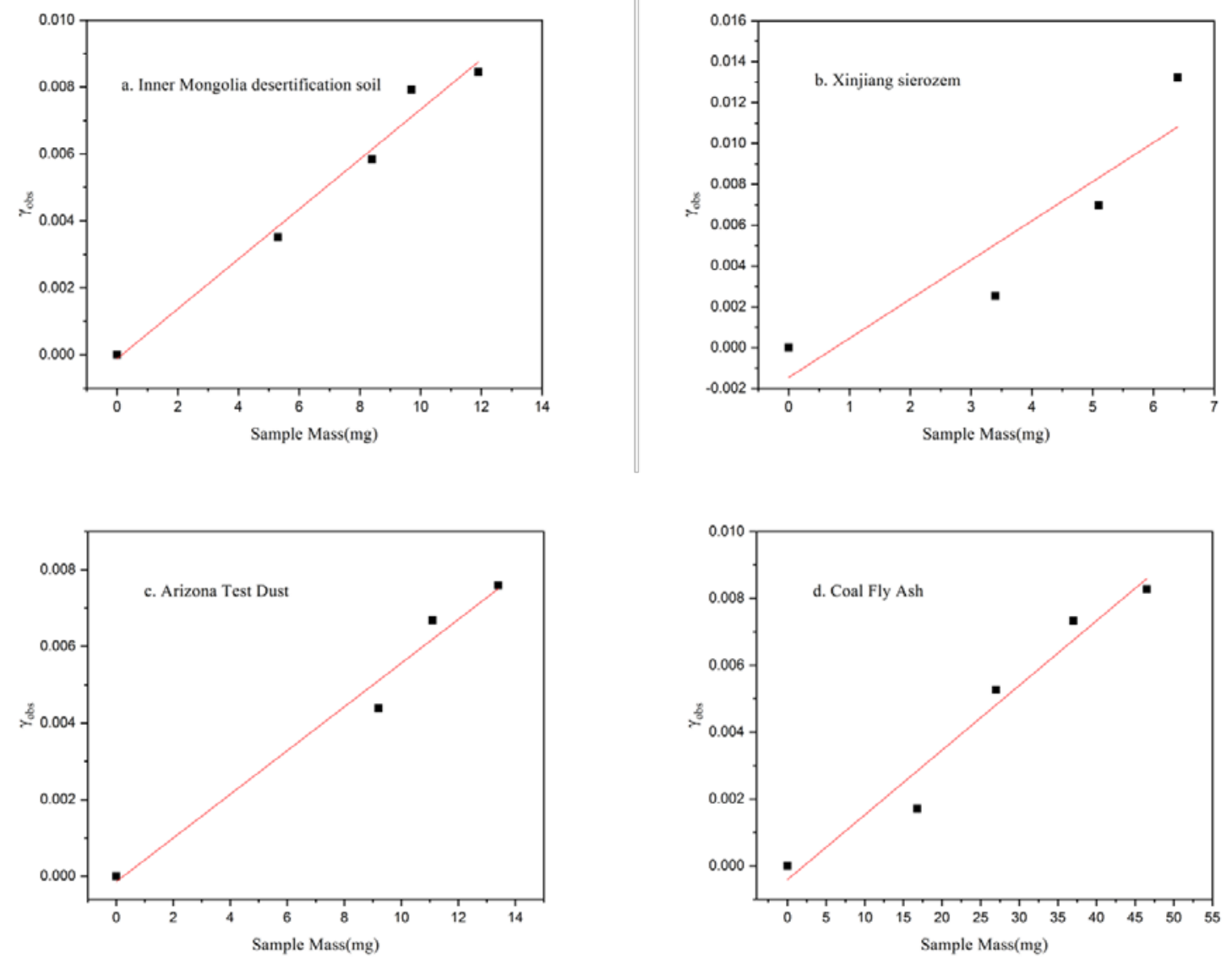

Figure S9. Linear mass-dependent regions of the observed uptake coefficient of acetic acid on a, Inner Mongolia desertification soil; b, Xinjiang sierozem mineral dust; c, Arizona Test Dust; d, Coal Fly Ash. 


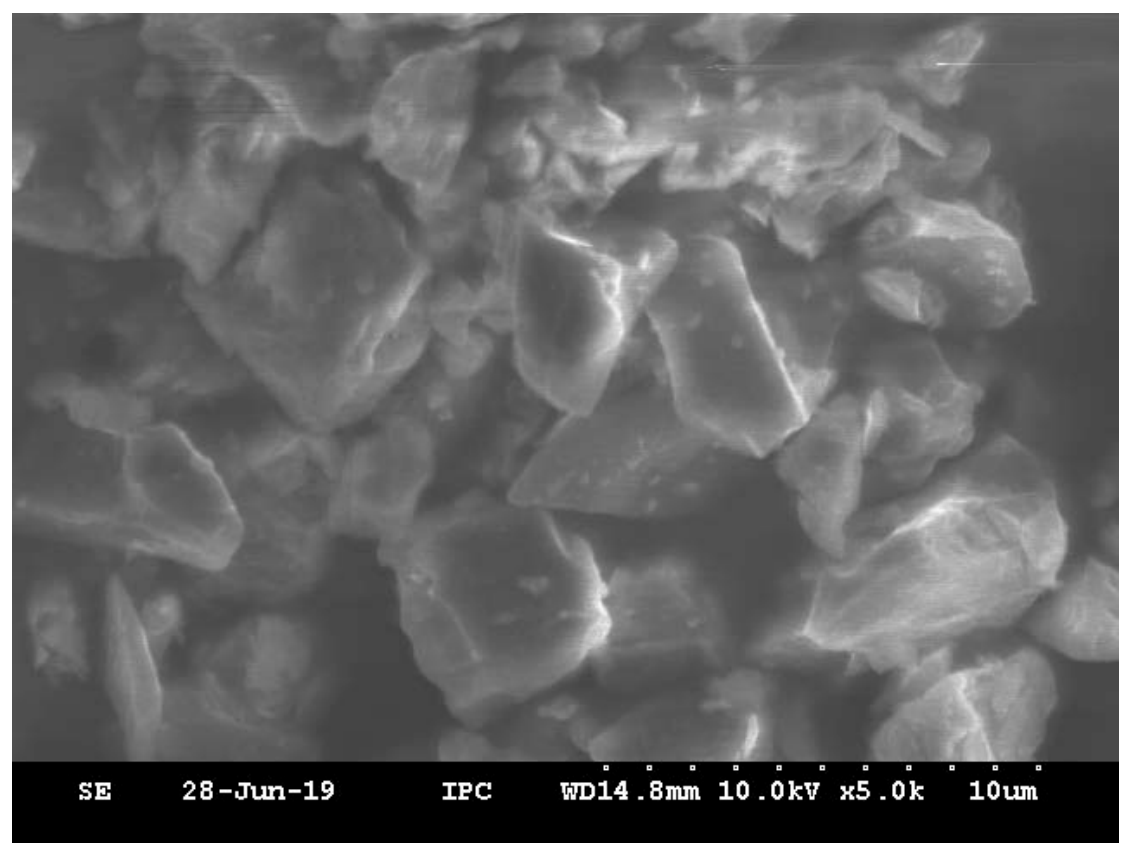

Figure S10. The SEM image of pure Arizona Test Dust using HITACHI S-4300 device.

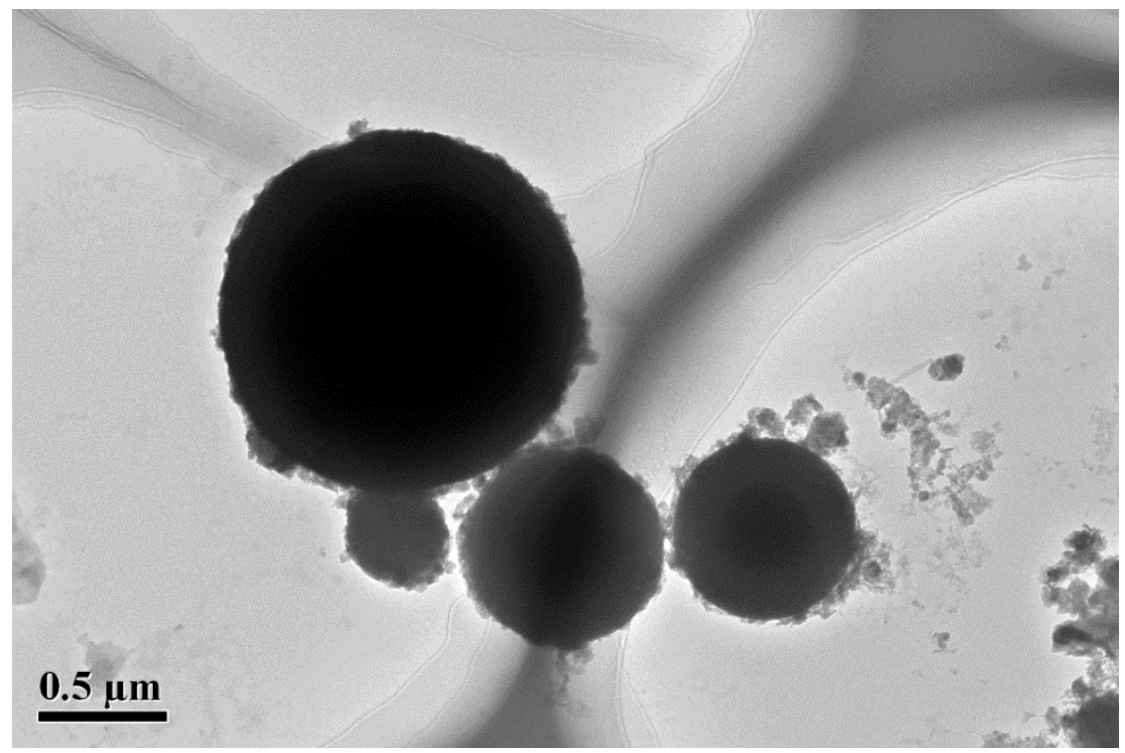

Figure S11. The TEM image of pure Coal Fly Ash using JEM-2100 device. 
Table S1. The element mass percentage of Inner Mongolia desertification, Xinjiang sierozem, Arizona Test Dust and Coal Fly Ash using XRF (XL 3t980)

\begin{tabular}{llllll}
\hline & \multicolumn{5}{c}{ Mass percentage } \\
\cline { 2 - 6 } Element & $\begin{array}{l}\text { Inner } \\
\text { Mongolia } \\
\text { desertificati } \\
\text { on }\end{array}$ & $\begin{array}{l}\text { Xinjiang } \\
\text { sierozem }\end{array}$ & $\begin{array}{l}\text { Arizona } \\
\text { Dust }\end{array}$ & $\begin{array}{l}\text { Test } \\
\text { Coal } \\
\text { Ash(USA) }\end{array}$ & Fly \\
\hline Fe & $7.46 \pm 0.14$ & $10.97 \pm 0.12$ & $9.70 \pm 0.13$ & $11.08 \pm 0.11$ \\
$\mathrm{Mn}$ & - & $0.20 \pm 0.02$ & $0.07 \pm 0.03$ & $0.05 \pm 0.02$ \\
$\mathrm{Ti}$ & $0.86 \pm 0.04$ & $1.29 \pm 0.04$ & $1.47 \pm 0.04$ & $1.50 \pm 0.05$ \\
$\mathrm{Ca}$ & $5.86 \pm 0.25$ & $18.79 \pm 0.31$ & $10.77 \pm 0.25$ & $17.08 \pm 0.26$ \\
$\mathrm{~K}$ & $8.30 \pm 0.2$ & $5.67 \pm 0.12$ & $4.55 \pm 0.11$ & $2.65 \pm 0.07$ \\
$\mathrm{Al}$ & $4.11 \pm 0.51$ & $10.04 \pm 0.54$ & $4.09 \pm 0.35$ & $19.00 \pm 0.56$ \\
$\mathrm{P}$ & - & - & $0.22 \pm 0.10$ & $1.59 \pm 0.08$ \\
$\mathrm{Si}$ & $73.42 \pm 0.84$ & $52.91 \pm 0.41$ & $69.10 \pm 0.56$ & $46.23 \pm 0.37$ & \\
$\mathrm{~S}$ & - & $0.11 \pm 0.03$ & - & $0.82 \pm 0.03$ \\
$\mathrm{Cl}$ & - & $0.03 \pm 0.01$ & $0.04 \pm 0.02$ & - \\
\hline
\end{tabular}

Table S2. The physical properties of formic acid and acetic acid.

\begin{tabular}{lllllll}
\hline Acids & CAS & Formula & Molecular & Fusion & $\begin{array}{l}\Delta_{\text {vap }} \mathrm{H}(298.15 \mathrm{~K}, \\
\mathrm{KJ} / \mathrm{mol})\end{array}$ & Dipole \\
& Number & & Weight(g/mol) & Point $\left({ }^{\circ} \mathrm{C}\right)$ & & moment(D) \\
\hline $\begin{array}{l}\text { Formic } \\
\text { Acid }\end{array}$ & $64-18-6$ & $\mathrm{CH}_{2} \mathrm{O}_{2}$ & 46 & 8.3 & 20.10 & $1.43 \pm 0.002$ \\
\hline $\begin{array}{l}\text { Acetic } \\
\text { Acid }\end{array}$ & $64-19-7$ & $\mathrm{C}_{2} \mathrm{H}_{3} \mathrm{C}_{2}$ & 60 & & & \\
\hline
\end{tabular}


Table S3 Correlation analysis of elements content with $\gamma_{\text {bet }}$ of formic acid.

\begin{tabular}{lcccccccc}
\hline \multirow{2}{*}{$\begin{array}{l}\gamma_{\text {bet. }} / \text { acid } \\
\text { acic }\end{array}$} & \multicolumn{2}{c}{$263 \mathrm{~K}$} & \multicolumn{2}{c}{$273 \mathrm{~K}$} & \multicolumn{2}{c}{$283 \mathrm{~K}$} & \multicolumn{2}{c}{$298 \mathrm{~K}$} \\
\cline { 2 - 9 } & Correl & $\mathrm{P}$ & Correl & $\mathrm{P}$ & Correl & $\mathrm{P}$ & Correl & $\mathrm{P}$ \\
\hline $\mathrm{Fe}$ & -0.93 & 0.07 & -0.93 & 0.07 & -0.94 & 0.06 & -0.89 & 0.11 \\
\hline $\mathrm{Ti}$ & -0.98 & 0.02 & -0.98 & 0.02 & -0.97 & 0.03 & -0.98 & 0.02 \\
\hline $\mathrm{Ca}$ & -0.80 & 0.21 & -0.79 & 0.21 & -0.82 & 0.18 & -0.74 & 0.26 \\
\hline $\mathrm{K}$ & 0.91 & 0.09 & 0.94 & 0.06 & 0.90 & 0.10 & 0.99 & 0.01 \\
\hline $\mathrm{Al}$ & -0.56 & 0.44 & -0.61 & 0.39 & -0.56 & 0.44 & -0.70 & 0.30 \\
\hline $\mathrm{Si}$ & 0.70 & 0.31 & 0.72 & 0.28 & 0.71 & 0.29 & 0.74 & 0.26 \\
\hline
\end{tabular}

Correl represent for the abbreviation for Pearson Correlation Coefficient, $\mathrm{P}$ is the result of significance test of correlation coefficient, $\mathrm{P}<0.05$ means that the correlation relationship is significant.

Table S4 Correlation analysis of element content with $\gamma_{\text {bet }}$ of acetic acid.

\begin{tabular}{lllllllll}
\hline \multirow{2}{*}{$\begin{array}{l}\gamma_{\text {bet }} \text { acid } \\
\text { lacetic }\end{array}$} & \multicolumn{2}{c}{$263 \mathrm{~K}$} & \multicolumn{2}{c}{$273 \mathrm{~K}$} & \multicolumn{2}{c}{$283 \mathrm{~K}$} & \multicolumn{2}{c}{$298 \mathrm{~K}$} \\
\cline { 2 - 9 } & Correl & $\mathrm{P}$ & Correl & $\mathrm{P}$ & Correl & $\mathrm{P}$ & Correl & $\mathrm{P}$ \\
\hline $\mathrm{Fe}$ & 0.79 & 0.21 & -0.66 & 0.34 & -0.96 & 0.04 & -0.90 & 0.10 \\
\hline $\mathrm{Ti}$ & 0.50 & 0.50 & -0.93 & 0.07 & -0.93 & 0.07 & -0.96 & 0.04 \\
\hline $\mathrm{Ca}$ & 0.86 & 0.14 & -0.45 & 0.55 & -0.87 & 0.13 & -0.77 & 0.23 \\
\hline $\mathrm{K}$ & -0.35 & 0.65 & 0.96 & 0.04 & 0.86 & 0.14 & 0.99 & 0.01 \\
\hline $\mathrm{Al}$ & 0.29 & 0.71 & -0.58 & 0.42 & -0.56 & 0.44 & -0.75 & 0.25 \\
\hline $\mathrm{Si}$ & -0.60 & 0.40 & 0.52 & 0.48 & 0.74 & 0.26 & 0.79 & 0.21 \\
\hline
\end{tabular}

Correl represent for the abbreviation for Pearson Correlation Coefficient, $\mathrm{P}$ is the result of significance test of the correlation coefficient, $\mathrm{P}<0.05$ means that the correlation relationship is significant. 
Table S5. The correlation coefficient between the uptake coefficient and Na mass fraction.

\begin{tabular}{lll}
\hline & \multicolumn{2}{l}{ Correlation coefficient } \\
\cline { 2 - 3 } Temperature/K & formic acid & acetic acid \\
\hline $298 / 293$ & 0.75 & 0.79 \\
283 & 0.69 & 0.71 \\
273 & 0.71 & 0.54 \\
263 & 0.68 & -0.54 \\
\hline
\end{tabular}

